\title{
REVIEW
}

\section{Risk of competitive sport in young athletes with heart disease}

\section{S Firoozi, S Sharma, W J McKenna}

Heart 2003;89:710-714

The majority of sudden deaths in young athletes occur in the context of underlying inherited or genetic cardiac disorders. The evaluation of every athlete regarding underlying cardiac disease is impractical and therefore needs to be targeted at those who are at a higher risk. A practical approach would be to channel efforts towards athletes with cardiac symptoms, those with a family history of inherited cardiac disease, and those with a family history of premature sudden death. There are potential pitfalls in the evaluation of young athletes using non-invasive tests when making the distinction between physiological adaptations to exercise and cardiac pathology. Physicians evaluating young athletes need to be aware of the spectrum of physiological adaptations and to be familiar with conditions responsible for sudden death in this population.

See end of article for authors' affiliations

Correspondence to: Professor W J McKenna, Department of

Cardiological Sciences, St George's Hospital Medical School, Cranmer Terrace, London SW17 ORE, UK wmckenna@sghms.ac.uk

Accepted

16 January 2003 n 490 вс, Pheidippides, the renowned Athenean long distance runner, suffered a sudden cardiac death (SCD) after running from the battlefield of Marathon to Athens to announce the great victory of the Greeks over the invaders. The sudden death of athletes remains a topic of interest among physicians and has led to the recognition of a number of cardiovascular disorders in this population. Approximately $80 \%$ of non-traumatic sudden deaths in young athletes are caused by inherited or congenital structural and functional cardiovascular abnormalities, which provide a substrate for arrhythmias predisposing to SCD. ${ }^{1-3}$ Hypertrophic cardiomyopathy (HCM) accounts for $40-50 \%$ of SCD in young athletes; table 1 summarises the other causes.

The precise incidence of cardiovascular related SCD in young athletes is unknown, as compre-

Table 1 Causes of sudden cardiac death in young athletes

Hypertrophic cardiomyopathy (responsible for up to $50 \%$ of cases)

Arrhythmogenic right ventricular cardiomyopathy

Congenital coronary artery anomalies

Premature coronary artery disease

Wolff-Parkinson-White syndrome

Long QT and Brugada syndromes

Idiopathic dilated cardiomyopathy

Myocarditis

Marfan's syndrome

Congenital aortic stenosis hensive data are lacking. SCD is thought to be uncommon, with estimates from the USA suggesting that 1 in 200000 competitors is affected. ${ }^{4}$ The true incidence is likely to be higher for a number of reasons. Firstly, the pathologist carrying out the postmortem examination in young athletes rarely has experience of the conditions involved and so subtle cases are missed. Furthermore, some conditions such as the ion channelopathies, which predispose to fatal arrhythmias, are not associated with structural heart disease and so the cause of death may remain unclear. Secondly, the lack of a national registry for SCD in athletes leads to an underestimate of SCD in athletes. There is reliance on media coverage, which focuses on higher profile athletes and on voluntary hospital reporting. In addition, retrospective data from referral centres regarding the cause of sudden death in young athletes is subject to bias depending on the particular expertise or area of interest of the institution. Thirdly, in the event of SCD in athletes the coroner's priority is to exclude foul play rather than to establish the precise cardiovascular diagnosis.

The majority $(>80 \%)$ of SCDs in young athletes occur either during or immediately after strenuous physical activity. ${ }^{5}$ This suggests that exercise may be a trigger for cardiac arrhythmias in those with certain cardiac disorders.

\section{ARGUMENTS FOR AND AGAINST CARDIOVASCULAR EVALUATION OF ATHLETES}

Physical exercise benefits cardiovascular health. ${ }^{6}$ In prospective epidemiological studies, exercise is consistently associated with a reduced risk of coronary artery disease and SCD. ${ }^{7-10}$ On the other hand, it is also recognised that a small but significant proportion of athletes die suddenly, possibly related to physical exercise as a trigger in the context of underlying cardiac disease, which provides the substrate for lethal arrhythmia. ${ }^{11}{ }^{12}$ Throughout history, society has made a special place for those few who are faster, stronger, and physically gifted. Sudden death in an athlete is tragic and highly publicised, especially when high profile athletes are involved, and raises concern among those in the community who regard athletes as the healthiest cohort of society.

Abbreviations: DCM, dilated cardiomyopathy; HCM, hypertrophic cardiomyopathy; LVH, left ventricular hypertrophy; peak $\dot{V}_{2}$, peak oxygen consumption rate; SCD, sudden cardiac death 
Ideally, all athletes would be evaluated for cardiovascular disease before athletic participation. However, the routine evaluation of all athletes is impractical because of the large numbers of people who need to be evaluated relative to the small potential positive yield. As well as not being cost effective, this approach would put a substantial burden on health care resources. On the other hand, athletes deserve to be evaluated to ensure that athletic participation is not a risk. Therefore, a common sense middle ground is sought by targeting high risk situations.

\section{ATHLETES WITH A DEFINITE DIAGNOSIS OF CARDIOVASCULAR DISEASE}

The 26th Bethesda conference was organised in January 1994 to formulate guidelines for participation in competitive sports for athletes with an identified cardiovascular abnormality. ${ }^{13}$ Experts in cardiovascular medicine and sports cardiology generated recommendations by means of consensus, and these provide the basis for physician advice to patients. The guidelines depend on the nature and severity of the cardiovascular abnormality and the classification of sport involved.

Athletes with unequivocal HCM should not participate in most competitive sports with the possible exception of sports with the lowest degree of intensity (for example, bowling, golf, or curling). ${ }^{14}$ This is regardless of the presence of symptoms and the magnitude of left ventricular hypertrophy (LVH) or left ventricular outflow tract obstruction. In view of the lower risk of sudden death in older HCM patients, individual judgement can be used in the absence of established risk markers for sudden death. These are: non-sustained ventricular tachycardia on Holter monitoring; a family history of premature (age $<40$ years) sudden death from HCM; a history of unexplained syncope; abnormal blood pressure response during upright exercise; severe LVH (maximal wall thickness $>30 \mathrm{~mm}$ ); and $>100 \mathrm{~mm} \mathrm{Hg}$ severe outflow tract obstruction.

With the advent of preclinical genetic diagnosis of HCM, a small number of young athletes may be identified to be gene positive but phenotypically be normal. The clinical significance of these findings will depend on the gene in question and will become clearer with better understanding of the genotype-phenotype relation. At present there is little evidence to preclude these athletes from taking part in competitive sports in the absence of symptoms or a family history of SCD. ${ }^{14}$

There are fewer data with regard to the relative risks of athletic training in athletes with the other cardiomyopathies such as arrhythmogenic right ventricular cardiomyopathy and idiopathic dilated cardiomyopathy (DCM). As a result, athletes with these conditions are advised not to participate in any competitive sports. ${ }^{14}$

Table 2 summarises the current recommendations for participation in competitive sports for athletes with other cardiovascular abnormalities.

\section{ATHLETES WITH FEATURES SUGGESTIVE OF CARDIAC DISEASE}

Cardiovascular evaluation in the young athlete should be directed at identifying conditions that may place the athlete at

Table 2 Current recommendations regarding athletic participation for athletes with cardiac conditions causing sudden death in young athletes

\begin{tabular}{|c|c|c|}
\hline Diagnosis & $\operatorname{Rec}$ & ommendation \\
\hline $\mathrm{HCM}$ & $\begin{array}{l}1 \\
2\end{array}$ & $\begin{array}{l}\text { Should not participate in most competitive sports with the possible exception of those of low intensity } \\
\text { Older athletes may participate depending on risk factor stratification }\end{array}$ \\
\hline ARVC & & Should not participate in competitive sports \\
\hline $\begin{array}{l}\text { Coronary artery } \\
\text { anomalies }\end{array}$ & $\begin{array}{l}1 \\
2\end{array}$ & $\begin{array}{l}\text { Should be excluded from competitive sports } \\
\text { Athletes without ischaemia on exercise stress testing may participate in sports }>6 \text { months after surgical treatment }\end{array}$ \\
\hline WPW & $\begin{array}{l}1 \\
2 \\
3\end{array}$ & $\begin{array}{l}\text { Athletes without structural heart disease, palpitations or tachycardia can participate in all competitive sports } \\
\text { Athletes with re-entrant tachycardia should be treated with radiofrequency ablation } \\
\text { Athletes with atrial flutter/fibrillation with slow accessory pathway conduction and no syncope can participate freely. Those } \\
\text { with syncope or fast accessory pathway conduction should be treated with radiofrequency ablation } \\
\text { Athletes with successful ablation of accessory pathway who are asymptomatic, have normal atrioventricular conduction on } \\
\text { electrophysiological study, and have no recurrence of tachycardia for 3-6 months can participate in all sports }\end{array}$ \\
\hline Ion channelopathies* & & Should not participate in competitive sports \\
\hline IDCM & & Should not participate in competitive sports \\
\hline $\begin{array}{l}\text { Premature coronary } \\
\text { artery disease }\end{array}$ & $\begin{array}{l}1 \\
2\end{array}$ & $\begin{array}{l}\text { If considered low riskt, can participate in low and moderate intensity sports. Should be re-evaluated annually } \\
\text { If considered to be at high riskt, may only participate in low intensity sports. Should be re-evaluated every } 6 \text { months }\end{array}$ \\
\hline Marfan's syndrome & 1 & $\begin{array}{l}\text { Athletes without a family history of premature sudden cardiac death and without aortic root dilatation can participate in low } \\
\text { and moderate intensity competitive sports. Serial } 6 \text { monthly monitoring of aortic root should be repeated } \\
\text { Athletes with aortic root dilatation can participate in low intensity sports only }\end{array}$ \\
\hline Myocarditis & $\begin{array}{l}1 \\
2\end{array}$ & $\begin{array}{l}\text { Should be withdrawn from competitive sports for about } 6 \text { months after onset of symptoms for convalescence } \\
\text { May return to competitive sports after normalisation of ventricular function and absence of clinically relevant arrhythmias on } \\
\text { ambulatory ECG monitoring }\end{array}$ \\
\hline Aortic stenosis & $\begin{array}{l}1 \\
2 \\
3 \\
4\end{array}$ & $\begin{array}{l}\text { Athletes with mild aortic stenosis ( }<20 \mathrm{~mm} \mathrm{Hg} \text { ) can participate in all competitive sports } \\
\text { Athletes with mild to moderate aortic stenosis ( } 21 \text { to } 40 \mathrm{~mm} \mathrm{Hg} \text { ) can participate in all low intensity sports. Some, depending } \\
\text { on exercise stress testing, can participate in low and moderate intensity sports } \\
\text { Athletes with severe aortic stenosis ( }>40 \mathrm{~mm} \mathrm{Hg} \text { ) or symptoms should not engage in any competitive sports } \\
\text { Athletes with bicuspid aortic valve, even without stenosis but with aortic dilatation, can participate in low intensity sports only. } \\
\text { Serial } 6 \text { monthly echocardiographic monitoring of aortic root and ascending aorta is recommended }\end{array}$ \\
\hline \multicolumn{3}{|c|}{$\begin{array}{l}\text { ARVC, arrhythmogenic right ventricular cardiomyopathy; HCM, hypertrophic cardiomyopathy; IDCM, idiopathic dilated cardiomyopathy; WPW, } \\
\text { Wolff-Parkinson-White syndrome. } \\
\text { *Long QT and Brugada syndromes. } \\
\text { †Low risk defined by normal systolic function, normal exercise tolerance for age, no ischaemia on exercise stress testing, no exercise induced complex } \\
\text { ventricular arrhythmia, and no haemodynamically significant coronary artery stenosis. }\end{array}$} \\
\hline
\end{tabular}


risk from sudden death. The basic components of evaluation are the clinical history and cardiac examination along with the resting ECG and the two dimensional transthoracic echocardiogram to look for structural and functional abnormalities.

In the case of HCM, the resting 12 lead ECG and two dimensional echocardiogram in particular are the ideal tests for diagnostic purposes. The diagnosis of HCM would depend on evidence of LVH in the absence of other causes. The majority of other conditions in question (for example, DCM, WolffParkinson-White syndrome, long QT syndrome, aortic stenosis) would also be diagnosed with this evaluation protocol. In some conditions, however, such as arrhythmogenic right ventricular cardiomyopathy, extra investigations such as ambulatory Holter monitoring, signal averaged ECG, and exercise testing are often required for diagnosis. Likewise, in the case of premature coronary disease or congenital coronary anomalies, exercise testing and myocardial perfusion imaging may often be necessary for diagnosis.

Given the time, expense, and potential psychosocial disruption from performing cardiovascular evaluation, it is reasonable to prioritise those athletes who can be defined as being at higher risk of having cardiac abnormalities. This would permit the channelling of finite financial and manpower resources to relevant target areas and would be the most efficient strategy. This can be achieved by evaluating specific groups of athletes.

\section{ATHLETES WITH SYMPTOMS}

\section{Syncope and palpitation}

Syncope is important and should not be dismissed as benign without further investigation. The exact circumstances need to be defined to see whether it is recurrent and whether it is exertion related or situational in nature. Its association with palpitations or warning signs should be sought to try to elucidate an underlying mechanism. Unexplained syncope in a young athlete in the context of exercise should be considered to be an aborted sudden death until proved otherwise.

The objective in evaluating an athlete complaining of palpitation is to define the likelihood of clinically significant arrhythmia.

Evaluation should be aimed at looking for structural heart disease and conduction abnormalities and should include 12 lead ECG, echocardiography, ambulatory ECG (Holter) monitoring, and tilt testing. If syncope is recurrent and preceded by palpitation or presyncope, a cardio-memo device can be used to monitor the cardiac rhythm. If syncope occurs infrequently, it may be necessary to implant a Reveal device to exclude arrhythmia as the cause. The significance of syncope or palpitations is influenced by the presence or absence of underlying cardiac disease.

\section{Dyspnoea on exertion}

Breathlessness on exertion is a difficult problem to quantify subjectively, particularly in athletes whose expectations regarding levels of exercise tolerance differ from those of the sedentary population. In addition to the ECG and echocardiogram (assessing left ventricular and valvar function), chest radiography and peak flow recording are useful. Such athletes should ideally be assessed by objective measurements of exercise capacity such as cardiopulmonary exercise testing with assessment of peak oxygen consumption rate (peak $\dot{\mathrm{V}}_{2}$ ), as well as submaximal indices of exercise capacity, which may also offer further insight into potential mechanisms of symptomatic limitation.

\section{Chest pain}

It is important to ascertain whether the pain is atypical in nature or whether it is caused by angina. Angina needs to be investigated further by echocardiography (to look for HCM, anomalous coronary arteries, and aortic stenosis), resting
ECG, exercise testing, and assessment of coronary artery disease risk factors. If doubt persists regarding an athlete with angina, myocardial perfusion imaging or coronary angiography may be indicated.

\section{ATHLETES WITH A FAMILY HISTORY OF INHERITED CARDIAC DISEASE}

Many of the conditions causing SCD in the young athlete are genetic with an autosomal dominant inheritance pattern. ${ }^{12}$ In general, when there is a known family history of an inherited cardiac disorder, the risk for patients developing the disease is one in two unless the penetrance of the mutation that causes the disease is low. If an athlete is known to have a family history of inherited cardiac disease, a thorough evaluation is warranted. The evaluation of these athletes would include a clinical history and examination, resting 12 lead ECG, and two dimensional echocardiography.

During evaluation of the athlete, symptoms such as exertional or postprandial chest pain, sustained palpitation, presyncope, or syncope should be sought, as in the context of a family history they are suggestive of an underlying cardiovascular abnormality. A detailed family history and pedigree to confirm the exact nature and pattern of inheritance of cardiac disease are important. The presence of premature (age $<40$ years) sudden death(s) in the family would be relevant, as it would indicate a malignant phenotype and have implications should the athlete under evaluation be affected. Clinical examination would be directed, depending on the family history, at outflow tract obstruction (in the case of HCM), aortic stenosis, sensorineural deafness (in the case of long QT syndrome), or the typical features of Marfan's syndrome. On analysing the resting ECG, typical features of the conditions in question are looked for, which table 3 summarises. The echocardiogram is carried out to look for structural heart disease including the cardiomyopathies and valvar abnormalities.

\section{ATHLETES WITH A FAMILY HISTORY OF UNEXPLAINED PREMATURE SUDDEN DEATH}

The presence of unexplained premature (age $<40$ years) sudden death in the family, particularly in parents or grandparents, means that the risk to the athlete in question potentially approaches one in two. Under these circumstances it is important to try to obtain as much information about the premature sudden death as possible. It would be important to try to get details of any necropsy reports or old medical notes. The following particular points need attention:

- heart weight, postmortem expert review of histology

- previous symptoms suggestive of cardiovascular disease or haemodynamic of electrical instability

- coronary artery disease risk factors.

The aim would be to attempt to create a probability of whether the premature sudden death is relevant to the athlete under evaluation and to try to establish the cause. The evaluation of the athlete would involve steps similar to those in an athlete with a known family history of cardiac disease.

\section{POTENTIAL DIFFICULTIES IN EVALUATION OF THE YOUNG ATHLETE AND THE DIFFERENTIATION OF ATHLETE'S HEART FROM PATHOLOGY}

Regular physical training leads to cardiovascular adaptations with both structural and functional changes, which are referred to as the "athlete's heart". These include an increase in left ventricular cavity size and wall thickness of $10-20 \%$ and an increase in left ventricular mass of up to $45 \% .{ }^{15-18}$ 
Table 3 Electrocardiographic changes seen in conditions causing sudden death in young athletes

\begin{tabular}{lll}
\hline Diagnosis & ECG changes \\
\hline HCM & 1 & Pathological Q waves \\
& 2 & ST segment flattening, depression, or both \\
& 4 & Pronounced ( $>0.2 \mathrm{mV}$ ) T wave inversion \\
& 1 & Teft axis deviation \\
ARVC & 2 & Ventricular extrasystoles with left bundle branch block morphology \\
& 3 & QS complexes in leads V1-3 \\
& 4 & QRS prolongation (<1 10 ms) in V1-3 \\
& 5 & Epsilon waves \\
WPW & 1 & Short PR interval \\
Long QT syndrome & 2 & Delta wave \\
& 1 & Prolonged QT and QTc intervals \\
Brugada syndrome & 3 & U waves \\
\hline \multirow{2}{*}{ QTc, QT interval corrected for heart rate. }
\end{tabular}

\section{LVH versus HCM}

In the majority of athletes LVH is mild and does not lead to absolute measurements exceeding normal limits. In a minority, however, LVH is quite pronounced and raises the differential diagnosis of HCM. This is confirmed by a large echocardiographic study of over 900 elite athletes, which showed that $2 \%$ had a maximal left ventricular wall thickness $>13 \mathrm{~mm} .{ }^{19}$ The distinction in this minority has important implications, as the diagnosis of HCM in an athlete is grounds for disqualification from competition in order to minimise the risk of sudden death. An incorrect diagnosis of HCM in an elite athlete may result in unnecessary withdrawal from competitive sport with adverse physical, financial, and psychological consequences.

Differentiation between physiological LVH and HCM is helped by characteristic echocardiographic features, which are derived from a number of studies (table 4). In general, HCM patients have localised hypertrophy with reduced cavity dimensions and evidence of impaired diastolic function, while athletes do not have segmental hypertrophy but do have normal or slightly increased left ventricular cavity dimensions and normal diastolic function. Furthermore, an athlete with significant LVH as a result of training alone would be expected to have supranormal metabolic exercise indices such as the peak $\dot{\mathrm{V}}_{2}$. Studies have shown that peak $\dot{\mathrm{V}}_{\mathrm{O}_{2}}$ and other indices of metabolic exercise are abnormal in most patients with HCM regardless of the magnitude of $\mathrm{LVH}^{20}{ }^{20}$ This provides a further tool for the differentiation between physiological $\mathrm{LVH}$ and HCM.

Some HCM gene carriers may have incomplete disease expression or an atypical phenotype with an abnormal ECG and a normal two dimensional echocardiogram. ${ }^{21} 22$ Such patients may be at increased risk of sudden death and present a difficulty in management, as correct diagnosis is important in terms of both prognosis and implications for familial evaluation. ${ }^{23}$ The concept that ECG abnormalities in the highly trained athlete are part of the spectrum of the athletic heart syndrome is misleading, and abnormal Q waves, left axis deviation, T wave inversion, and ST depression should lead to serious consideration of underlying cardiac abnormality.

\section{Left ventricular cavity dilatation versus DCM}

Regular intensive physical exercise can lead to an increase in cardiac dimensions including left and right ventricular cavity enlargement. ${ }^{16}$ In a significant proportion (30\%) of athletes the left ventricular cavity size exceeds the upper limits of normal and in a minority the magnitude of left ventricular cavity enlargement is comparable with that encountered in some patients with DCM. ${ }^{18}$ The systolic function in the majority of cases of physiological left ventricular dilatation is normal and therefore assists in the differentiation from DCM. In some athletes, however, the ejection fraction at rest may be subnormal because of a large end diastolic cavity size, yet exercise capacity is excellent due to augmentation of the ejection fraction upon exercise. Furthermore, unlike in HCM, the ECG has a very low sensitivity and specificity for the diagnosis of DCM. As a result, evaluating the athlete by ECG and echocardiography may not differentiate between physiological and pathological left ventricular dilatation.

Under these circumstances the presence of symptoms associated with fluid congestion, palpitations or syncope, a positive family history of DCM, or a subnormal cardiopulmonary exercise test result would help in the distinction between athlete's heart and DCM.

\section{REPOLARISATION CHANGES VERSUS ION CHANNELOPATHIES}

Minor repolarisation abnormalities, including mild elevation of the J point associated with mild ST segment elevation in the

Table 4 Echocardiographic features of athlete's heart and HCM

\begin{tabular}{lll}
\hline & Athlete's heart & HCM \\
\hline Maximal left ventricular wall thickness $(\mathrm{mm})$ & $<16$ & $\geqslant 16$ \\
LVH pattern & Concentric & ASH/variable \\
Left ventricular cavity size & Large & Small \\
Diastolic function & Normal & Impaired \\
Left atrial size & Normal & Dilated \\
\hline \multirow{2}{*}{ ASH, asymmetrical septal hypertrophy; LVH, Left ventricular hypertrophy. }
\end{tabular}


anterior chest leads and mild T wave inversion $(<0.2 \mathrm{mV})$ in VI-2 and the inferior leads, are a frequent finding in young athletes. A significant proportion of young athletes also possess minor intraventricular conduction abnormalities such as incomplete right bundle branch block.

With better understanding of the long QT and Brugada syndromes, it is becoming clearer that in some cases of these conditions, repolarisation changes similar to those commonly seen in young athletes are present. For example, it is recognised that in some subtypes of the long QT syndrome, abnormalities of $\mathrm{T}$ wave morphology, $\mathrm{T}$ wave microalternans, and small $U$ waves in the proximity of the terminal portion of the $\mathrm{T}$ wave are present. ${ }^{24}$ Similarly, in the Brugada syndrome, the characteristic electrocardiographic abnormality is the presence of ST segment elevation along with right bundle branch block in the anterior leads. ${ }^{25}$ These features raise the possibility of misdiagnosing normality in a young athlete and allowing competitive participation in the face of a potentially lethal cardiac disorder. Furthermore, both the long QT and Brugada syndromes do express incomplete penetrance ${ }^{25}$ with latent ECG changes, and therefore relying on the ECG for their diagnosis would lead to false negatives. In some other cases the ECG changes may be subtle and there may be a need for provocation tests to confirm the diagnosis. These difficulties are highlighted by the relative lack of exposure and consequent unfamiliarity with these conditions in most cardiologists' daily practice.

In cases of uncertainty, the presence of a history of syncope or a family history of premature sudden death should raise the level of suspicion and lead to expert help being sought.

\section{SUMMARY}

Resources are not available to evaluate all young athletes before participation in competitive sports. As a result the cardiovascular evaluation of young athletes needs to be targeted at high risk areas and focus on those who are at greatest risk and those with symptoms and a family history of premature cardiac disease or sudden death. The involvement of amateur and professional sporting bodies and organisations with the above approach would improve the safety of athletic participation. The 12 lead ECG is the most sensitive practical tool for identification of underlying cardiac abnormalities.

\section{Authors' affiliations}

S Firoozi, S Sharma, W J McKenna, Department of Cardiological Sciences, St George's Hospital Medical School, London, UK

\section{REFERENCES}

1 Maron BJ, Epstein SE, Roberts WC. Causes of sudden death in competitive athletes. J Am Coll Cardiol 1986;7:204-14.

2 Corrado D, Thiene G, Nava A, et al. Sudden death in young competitive athletes: clinicopathologic correlations in 22 cases. Am J Med 1990;89:588-96.
3 Opie LH. Sudden death and sport. Lancet 1975;i:263-6.

4 Epstein SE, Maron BJ. Sudden death and the competitive athlete: perspectives on preparticipation screening studies. J Am Coll Cardiol 1986; 7:220-30.

5 Maron BJ, Shirani J, Poliac LC, et al. Sudden death in young competitive athletes. Clinical, demographic, and pathological profiles. JAMA 1996;276:199-204.

6 Paffenbarger RS Jr, Hyde RT, Wing AL, et al. The association of changes in physical-activity level and other lifestyle characteristics with mortality among men. N Engl J Med 1993;328:538-45.

7 Slattery ML, Jacobs DR Jr, Nichaman MZ. Leisure time physical activity and coronary heart disease death. The US Railroad Study. Circulation 1989;79:304-11

8 Morris JN, Everitt MG, Pollard R, et al. Vigorous exercise in leisure-time: protection against coronary heart disease. Lancet 1980;ii:1207-10.

9 Leon AS, Connett J, Jacobs DR Jr, et al. Leisure-time physical activity levels and risk of coronary heart disease and death. The multiple risk factor intervention trial. JAMA 1987;258:2388-95.

10 Ekelund LG, Haskell WL, Johnson JL, et al. Physical fitness as a predictor of cardiovascular mortality in asymptomatic North American men. The lipid research clinics mortality follow-up study. $N$ Engl J Med 1988;319:1379-84.

11 Maron BJ. Sudden death in young athletes. Lessons from the Hank Gathers affair. N Engl J Med 1993;329:55-7.

12 Maron BJ, Roberts WC, McAllister HA, et al. Sudden death in young athletes. Circulation 1980;62:218-29.

13 Anon. 26th Bethesda Conference: recommendations for determining eligibility for competition in athletes with cardiovascular abnormalities. January 6-7, 1994. J Am Coll Cardiol 1994;24:845-99.

14 Maron BJ, Isner JM, McKenna WJ. 26th Bethesda conference: recommendations for determining eligibility for competition in athletes with cardiovascular abnormalities. Task force 3: hypertrophic cardiomyopathy, myocarditis and other myopericardial diseases and mitral valve prolapse. J Am Coll Cardiol 1994;24:880-5

15 Maron BJ. Structural features of the athlete heart as defined by echocardiography. J Am Coll Cardiol 1986;7:190-203.

16 Huston TP, Puffer JC, Rodney WM. The athletic heart syndrome. N Engl J Med 1985;313:24-32

17 Fagard R, Aubert A, Lysens R, et al. Noninvasive assessment of seasonal variations in cardiac structure and function in cyclists. Circulation 1983;67:896-901.

18 Spirito P, Pelliccia A, Proschan MA, et al. Morphology of the "athlete's heart" assessed by echocardiography in 947 elite athletes representing 27 sports. Am J Cardiol 1994;74:802-6.

19 Pelliccia A, Maron BJ, Spataro A, et al. The upper limit of physiologic cardiac hypertrophy in highly trained elite athletes. N Engl J Med 1991;324:295-301.

20 Sharma $S$, Elliott $P$, Whyte $G$, et al. Utility of cardiopulmonary exercise in the assessment of clinical determinants of functional capacity in hypertrophic cardiomyopathy. Am J Cardiol 2000;86:162-8.

21 Thierfelder L, Watkins H, MacRae C, et al. Alpha-tropomyosin and cardiac troponin T mutations cause familial hypertrophic cardiomyopathy: a disease of the sarcomere. Cell 1994;77:701-12.

22 McKenna WJ, Stewart JT, Nihoyannopoulos P, et al. Hypertrophic cardiomyopathy without hypertrophy: two families with myocardial disarray in the absence of increased myocardial mass. Br Heart $J$ 1990;63:287-90.

23 Spirito $\mathbf{P}$, Seidman CE, McKenna WJ, et al. The management of hypertrophic cardiomyopathy. N Engl J Med 1997;336:775-85.

24 Zhang L, Timothy KW, Vincent GM, et al. Spectrum of ST-T-wave patterns and repolarization parameters in congenital long-QT syndrome: ECG findings identify genotypes. Circulation 2000;102:2849-55.

25 Brugada P, Brugada J. Right bundle branch block, persistent ST segment elevation and sudden cardiac death: a distinct clinical and electrocardiographic syndrome. A multicenter report. J Am Coll Cardiol 1992;20:1391-6. 